\title{
Comparative Studies on Generation of Stair Step Radiation Patterns
}

\author{
M.Chandrasekhar ${ }^{1}$, G.S.N.Raju ${ }^{2}$ \\ ${ }^{1 .}$ Research Scholar, Dept. of Electronics and Communication Engineering, College of Engineering (A), Andhra \\ University, Visakhapatnam, Andhra Pradesh, India \\ 2. Professor, Dept. of Electronics and Communication Engineering, College of Engineering (A), Andhra \\ University, Visakhapatnam, Andhra Pradesh, India
}

\begin{abstract}
Antenna array synthesis is usually done by controlling amplitude and phase levels or element spacing functions of the array to get the desired characteristics of the radiation pattern. In this paper, design of equispaced linear array of dipoles is presented. A new amplitude distribution is proposed for generation of stair step patterns with zero phase. These patterns are produced from dipole elements using Fourier transform method. The goal of the work is to compensate mutual coupling effects of a linear dipole array. The computed patterns are compared with those of the isotropic elements for different number of elements. The deviation in the patterns of array of isotropic radiators and the array of practical element is found to be minimum. These patterns are used to identify multiple targets moving in different altitudes and different angular regions.
\end{abstract}

Keywords: Luneburg Lens antenna, Geometrical optics, Dipole elements, Broad beam, Ripples, Fourier Transform method.

\section{Introduction}

In recent years, there has been considerable interest in designing antenna arrays with broad beam patterns. The aim of the antenna synthesis is to find the appropriate excitation coefficients of the elements for the generation of desired radiation pattern [1]. To get desired radiation pattern of the antenna, different types of synthesis methods are used depending on their usability and the convenience in the situation under consideration.

It's the geometry of the aperture and the distribution of the phase and amplitude of excitation across the aperture mainly govern the final pattern of the antenna. In arrays the element pattern is also a controlling parameter [2-3] and the number of elements within a specified aperture has a direct influence on the final pattern.

Desired beam shape can be generated from well shaped reflector antennas. The principle involved in beam shaping can be easily explained in terms of geometrical optics. Its advantage is simplicity in design and fabrication [4-6]. However, it is able to do only very limited beam scanning and wide beam scanning is mostly achieved by mechanical rotation, which needs a powerful motor when the reflector is large and it is expensive. In such case , an attractive alternative is to use a Luneburg lens which offers scan capability over a very wide angular range.

It has been used in a variety of antenna and scattering applications. In antenna applications, the main advantages of lenses are the ability to form simultaneous beams in various directions with equal gain and their broad band behaviour .Recent advances in material technology have enabled the manufacturing of large light weight lenses with the desired electrical permittivity. Moreover, it has lower reflection at the lens surface.

Geometrical optics predicts 100percent efficiency regardless of the feed array size. The actual feed size can be determined from simple diffraction concepts[7]. The geometrical optics aperture distribution is a useful distribution with no spill over. The spill over results in the loss of gain, phase error and amplitude ripples.

Giorgio .V. Borgiotti [8] described a radiating structure to generate shaped beams which consists of a bootlace lens with linear outer and circular inner profiles resulting excellent scan performance over moderate frequency band. Luneburg lens antennas are used in multiple beam scanning applications. Basically, a Luneburg lens transforms a point source radiation into a plane wave by correcting the phase of the source. Since the main principle of the Luneburg lens is based on the geometric optics concepts[9] and it is operated in broad band frequency range. Also, the spherical symmetry of the lens allows multiple beams scanning by mean of an array of feeds placed around the lens surface. Arrays with sector patterns can be used as feeds in reflector or lens antennas for providing high aperture efficiency with minimum spill over. The scatter field of the lens antenna is determined by using an analytic technique is based on the Green's function of the multi-layered spherical shell .Due to these excellent features, lens antennas are used more extensively in radars, military and satellite communications. 
The aim of these studies has been the reduction of the number of costly control elements, phase shifters or variable power dividers as compared to the number in a phased array designed in a conventional way. Stochastic methods, such as GA, PSO, Firefly and DE which can escape from local optimization solutions. The stochastic methods can solve large optimization problems, but the computation time is huge and will grow rapidly with the problem size. In this paper, Fourier transform method is applied for realization of stair - step pattern [10]. This method can solve large array synthesis with much less time compared with the stochastic methods.

\section{Analysis}

In this paper, the radiating elements in the array of present interest are considered to be point sources and dipole elements, optical system is described which satisfies the conditions

a) Aperture efficiency $=100 \%$ for all scan angles

b) Number of active elements $=$ Number of beam widths of scan

and provides nearly ideal performance comparable to a standard constrained system. The geometry is described in terms of geometric optics where it is shown that a generalized Luneburg Lens is a necessary element [9] in the ptical system .Performance can be analyzed for large optical elements using simplified corrections for diffraction effects.

The Luneburg Lens is a spherically symmetric delay-type lens formed of a dielectric with index of refraction ' $n$ ' which varies as a function of radius, as given by

$$
\mathrm{n}=\sqrt{\varepsilon_{\mathrm{r}}}=\sqrt{2-\left(\frac{\mathrm{r}}{\mathrm{R}}\right)^{2}}
$$

Here, $r=$ radial distance from centre of sphere and

$\mathrm{R}=$ radius of the sphere.

A Luneburg lens is fabricated using the dielectric material such as polystyrene or polyethylene. These dielectric materials have dielectric losses. At higher frequencies, the dielectric loss will be greater .The effect of dielectric loss is less than effect of air gaps if low loss dielectric material is used for lens fabrication. However, the gain loss cannot be ignored at higher operating frequencies.

Air - gaps between spherical shells may be unavoidable in the process of constructing the Luneburg lens with a finite number of spherical shells. The air -gaps affect the performance of the Luneburg lens antenna [11].

Consider a circular lens shown in fig1. Its dielectric constant depends on radial distance. Assume that this lens acts as corrective lens of the system. Also assume that the lens bends the rays coming from a point source placed on the lens surface at radial distance ' $d$ ' to another point at distance $\mathrm{D}>\mathrm{d}$. The source, centre of the lens and focal point lie along same straight line .If the above focal condition is true for line pair of points then all points on the circular lens surface will image to unique points on the image surface,radiusD.The distribution of these image sources will be a stretched replica of the source distribution .These sources on the image circle are again mapped to a linear aperture without distortion by means of equal line lengths connecting all point pairs whose arc lengths measured from the line of symmetry are the same.

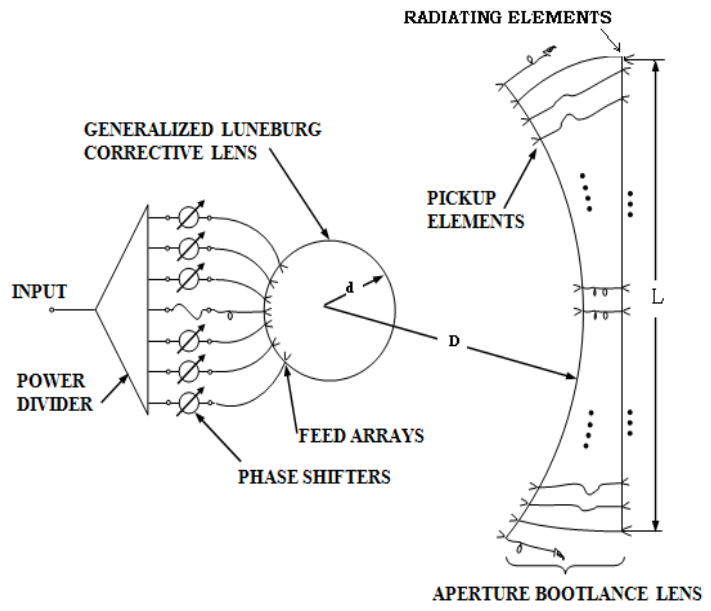

Fig1. Major Components of Optical Antenna System[9]. 
The Bootlace aperture lens used in this system is not the commonly used Abbe or Rotman lens[12]. Only broadside incidence will focus all the rays to a single point. At maximum scan all incoming rays are tangent to the lens. This gives maximum scan angle and usable D/L ratio. The portion of the lens which will be illuminated on receive must not overlap the feed array.

In general, there exist diffraction effects. These can be obtained by adopting the sub array view point and by applying a mixture of geometrical optics and diffraction theory to estimate the aperture distribution [13]. A spacing of $\lambda / 2$ of radiating elements are assumed to be matched for all angles and to have a cosine type of element pattern as in the case of large array. A similar array with spacing $\mathrm{S}$ is placed on the inner feed of the aperture lens. One element in the feed array is excited with unit power. The distribution in a reference circle of radius (D-d) cantered on the receiving element located on the geometrical focus is obtained using geometrical optics. It is used to generate stair step type of beams. It is well known that the stair step types of beams are widely required to detect multi level targets. This method is also extend for practical radiating elements, dipoles of 21 and 61 elements.

Antenna array generally consists of identical antennas. This is not necessary but it is more practical, Simple and convenient for design and fabrication because each identical antenna will have identical radiation characteristics and impedances [14]. The antenna elements may be any type wire, dipole, loop, aperture etc.

Dipoles of finite length are widely used as simple antennas and as radiating elements in arrays. Dipole is antenna composed of simple radiating element split into two sections and fed into the split. Not all dipoles are split and fed in the center because currents can be excited on it electromagnetically or it can be center fed. For a short dipole the length is less than $\lambda / 2$ and the self-impedance is generally capacitive. For half wave dipole the length is generally $\lambda / 2$ and the self impedance of $40-70 \mathrm{ohms}$ with no reactive component and provides good match for coaxial cables [15-16]. The self-impedance of dipole varies with its length and height above the ground. It is possible to use a center fed dipole over a wide range of frequencies by feeding it with low pass transmission line and providing impedance matching. The significant advantage as regards bandwidth with respect to pattern quality in the presence of a backing ground plane and mainly concerned with the mechanisms contributing to the scattering and the resulting fields and their patterns. The radiation pattern of dipole array is computed by taking element pattern of dipole into account.

Case: I

\section{Formulation}

For sector beam represented by an expression of the form

$\mathrm{F}(\mathrm{u})=1, \quad-\mathrm{u}_{0} \leq \mathrm{u} \leq \mathrm{u}_{0}$

$$
=0 \text {, otherwise }
$$

For an odd number of elements $\mathrm{N}=2 \mathrm{M}+1$, the normalised excitation coefficients can be obtained by the Fourier formula .

$$
A_{n}=\frac{1}{2} \int_{-u_{0}}^{u_{0}} E(u) e^{-j n \pi u} d u
$$

In the present work, the stair -step pattern represented by an expression of the form.

For three stair -steps

$$
\begin{aligned}
\mathrm{F}(\mathrm{u}) & =\frac{1}{3}, & & \frac{-3}{3} \leq \mathrm{u} \leq \frac{-2}{3} \\
& =\frac{2}{3}, & & \frac{-2}{3} \leq \mathrm{u} \leq \frac{-1}{3} \\
& =\frac{3}{3}, & & \frac{-1}{3} \leq \mathrm{u} \leq \frac{1}{3} \\
& =\frac{2}{3}, & & \frac{1}{3} \leq \mathrm{u} \leq \frac{2}{3} \\
& =\frac{1}{3}, & & \frac{2}{3} \leq \mathrm{u} \leq \frac{3}{3}
\end{aligned}
$$


For four stair-steps

$$
\begin{array}{rlrl}
\mathrm{F}(\mathrm{u}) & =\frac{1}{4}, & & \frac{-4}{4} \leq \mathrm{u} \leq \frac{-3}{4} \\
& =\frac{2}{4}, & \frac{-3}{4} \leq \mathrm{u} \leq \frac{-2}{4} \\
& =\frac{3}{4}, & \frac{-2}{4} \leq \mathrm{u} \leq \frac{-1}{4} \\
& =\frac{4}{4}, & \frac{-1}{4} \leq \mathrm{u} \leq \frac{1}{4} \\
& =\frac{3}{4}, & \frac{1}{4} \leq \mathrm{u} \leq \frac{2}{4} \\
& =\frac{2}{4}, & \frac{2}{4} \leq \mathrm{u} \leq \frac{3}{4} \\
& =\frac{1}{4}, & \frac{3}{4} \leq \mathrm{u} \leq \frac{4}{4}
\end{array}
$$

For five stair-steps

$$
\begin{aligned}
\mathrm{F}(\mathrm{u}) & =\frac{1}{5}, & & \frac{-5}{5} \leq \mathrm{u} \leq \frac{-4}{5} \\
& =\frac{2}{5}, & & \frac{-4}{5} \leq \mathrm{u} \leq \frac{-3}{5} \\
& =\frac{3}{5}, & & \frac{-3}{5} \leq \mathrm{u} \leq \frac{-2}{5} \\
& =\frac{4}{5}, & & \frac{-2}{5} \leq \mathrm{u} \leq \frac{-1}{5} \\
& =\frac{5}{5}, & & \frac{-1}{5} \leq \mathrm{u} \leq \frac{1}{5} \\
& =\frac{4}{5}, & & \frac{1}{5} \leq \mathrm{u} \leq \frac{2}{5} \\
& =\frac{3}{5}, & & \frac{2}{5} \leq \mathrm{u} \leq \frac{3}{5} \\
& =\frac{2}{5}, & & \frac{3}{5} \leq \mathrm{u} \leq \frac{4}{5} \\
& =\frac{1}{5}, & & \frac{4}{5} \leq \mathrm{u} \leq \frac{5}{5}
\end{aligned}
$$

For three stair-steps

$$
A_{n}=\frac{1}{2}\left[\int_{\frac{-3}{3}}^{\frac{-2}{3}} \frac{1}{3} e^{-j n \pi u} d u+\int_{\frac{-2}{3}}^{\frac{-1}{3}} \frac{2}{3} e^{-j n \pi u} d u+\int_{\frac{-1}{3}}^{\frac{1}{3}} \frac{3}{3} e^{-j n \pi u} d u+\int_{\frac{1}{3}}^{\frac{2}{3}} \frac{2}{3} e^{-j n \pi u} d u+\int_{\frac{2}{3}}^{\frac{3}{3}} \frac{1}{3} e^{-j n \pi u} d u\right]
$$

After integration and simplification, we get

$$
\mathrm{A}_{\mathrm{n}}=\frac{-2}{9} \frac{\sin \left(\mathrm{n} \pi \frac{2}{3}\right)}{\mathrm{n} \pi \frac{2}{3}}+\frac{1}{3} \frac{\sin (\mathrm{n} \pi)}{\mathrm{n} \pi}-\frac{2}{9} \frac{\sin \left(\mathrm{n} \frac{\pi}{3}\right)}{\mathrm{n} \frac{\pi}{3}}+\frac{4}{9} \frac{\sin \left(\mathrm{n} \pi \frac{2}{3}\right)}{\mathrm{n} \pi \frac{2}{3}}+\frac{1}{3} \frac{\sin \left(\mathrm{n} \frac{\pi}{3}\right)}{\mathrm{n} \frac{\pi}{3}}
$$

For four stair-steps 


$$
A_{n}=\frac{1}{2}\left[\begin{array}{l}
\int_{\frac{-4}{4}}^{\frac{-3}{4}} \frac{1}{4} e^{-j n \pi u} d u+\int_{\frac{-3}{4}}^{\frac{-2}{4}} \frac{2}{4} e^{-j n \pi u} d u+\int_{\frac{-2}{4}}^{\frac{3}{4}} \frac{3}{4} e^{-j n \pi u} d u+\int_{-\frac{1}{4}}^{\frac{1}{4}} \frac{4}{4} e^{-j n \pi u} d u+ \\
\frac{2}{4} \frac{3}{\frac{3}{4}} e^{-j n \pi u} d u+\int_{\frac{2}{4}}^{\frac{2}{4}} \frac{2}{4} e^{-j n \pi u} d u+\int_{\frac{3}{4}}^{\frac{1}{4}} \frac{1}{4} e^{-j n \pi u} d u
\end{array}\right]
$$

After integration and simplification, we get

$$
\begin{aligned}
A_{n}= & \frac{-3}{16} \frac{\sin \left(n \pi \frac{3}{4}\right)}{n \pi \frac{3}{4}}+\frac{1}{4} \frac{\sin (n \pi)}{n \pi}-\frac{4}{16} \frac{\sin \left(n \pi \frac{2}{4}\right)}{n \pi \frac{2}{4}}+\frac{6}{16} \frac{\sin \left(n \pi \frac{3}{4}\right)}{n \pi \frac{3}{4}} \\
& -\frac{3}{16} \frac{\sin \left(n \pi \frac{1}{4}\right)}{n \pi \frac{1}{4}}+\frac{6}{16} \frac{\sin \left(n \pi \frac{2}{4}\right)}{n \pi \frac{2}{4}}+\frac{1}{4} \frac{\sin \left(n \pi \frac{1}{4}\right)}{n \pi \frac{1}{4}}
\end{aligned}
$$

For five stair - steps,

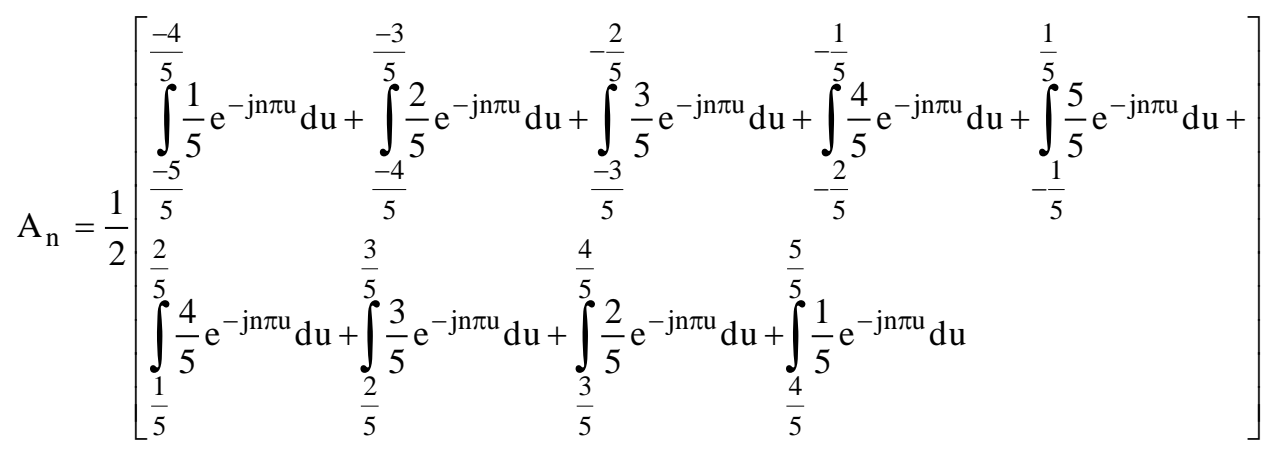

After integration and simplification, we get

$$
\begin{aligned}
& A_{n}=\frac{-4}{25} \frac{\sin \left(n \pi \frac{4}{5}\right)}{n \pi \frac{4}{5}}+\frac{1}{5} \frac{\sin (n \pi)}{n \pi}-\frac{6}{25} \frac{\sin \left(n \pi \frac{3}{5}\right)}{\mathrm{n} \pi \frac{3}{5}}+\frac{8}{25} \frac{\sin \left(\mathrm{n} \pi \frac{4}{5}\right)}{\mathrm{n} \pi \frac{4}{5}}-\frac{6}{25} \frac{\sin \left(\mathrm{n} \pi \frac{2}{5}\right)}{\mathrm{n} \pi \frac{2}{5}} \\
& +\frac{9}{25} \frac{\sin \left(\mathrm{n} \pi \frac{3}{5}\right)}{\mathrm{n} \pi \frac{3}{5}}-\frac{4}{25} \frac{\sin \left(\mathrm{n} \pi \frac{1}{5}\right)}{\mathrm{n} \pi \frac{1}{5}}+\frac{8}{25} \frac{\sin \left(\mathrm{n} \pi \frac{2}{5}\right)}{\mathrm{n} \pi \frac{2}{5}}+\frac{1}{25} \frac{\sin \left(\mathrm{n} \pi \frac{1}{5}\right)}{\mathrm{n} \pi \frac{1}{5}}
\end{aligned}
$$

When the radiating elements are isotropic in nature, the transmit pattern $E(u)$ of a distribution $A_{n}$ on the inner array of the aperture lens is given by

$$
E(u)=\left(\frac{k s}{2 \pi} \sqrt{1-u^{2}}\right)^{\frac{1}{2}} \sum_{n} A_{n} e^{j k n s u}
$$

For an odd number of elements $\mathrm{N}=2 \mathrm{M}+1$, the elements are placed at

$\mathrm{x}_{\mathrm{n}}=\mathrm{ns}, \mathrm{n}=0, \pm 1, \pm 2 \ldots \pm \mathrm{N}$

Here,

$\mathrm{u}=\sin \theta$

$\mathrm{A}_{\mathrm{n}}=$ Amplitude excitation of $\mathrm{n}^{\text {th }}$ element,

$\mathrm{s}=$ element spacing,

$\mathrm{n}=$ zero for a element centred on the geometric focus.

Case: II 
The normalized radiation field of half wave dipole is given by

$\mathrm{E}_{\mathrm{p}}=\left[\frac{\cos \left(\frac{\mathrm{kL}}{2} \cos \theta\right)-\cos \left(\frac{\mathrm{kL}}{2}\right)}{\sin \theta}\right]$

For the non-isotropic radiating element, the resultant pattern is given by

$E(u)=E_{p} \times\left(\frac{k s}{2 \pi} \sqrt{1-u^{2}}\right)^{\frac{1}{2}} \sum_{n} A_{n} e^{j k n s u}$

Here,

$\mathrm{E}_{\mathrm{p}}=$ Element Pattern.

\section{Simulation Result}

Using the equations (4-6), the variation of source distribution is presented in figures $(2,4,6,8,10$ and 12) and corresponding excitation tables are given for 21 and 61 elements. Introducing the excitation so obtained, radiation patterns are numerically computed using MATLAB software for array of dipoles and isotropic elements and which are compared shown in figures(3,5,7,9,11 and13).

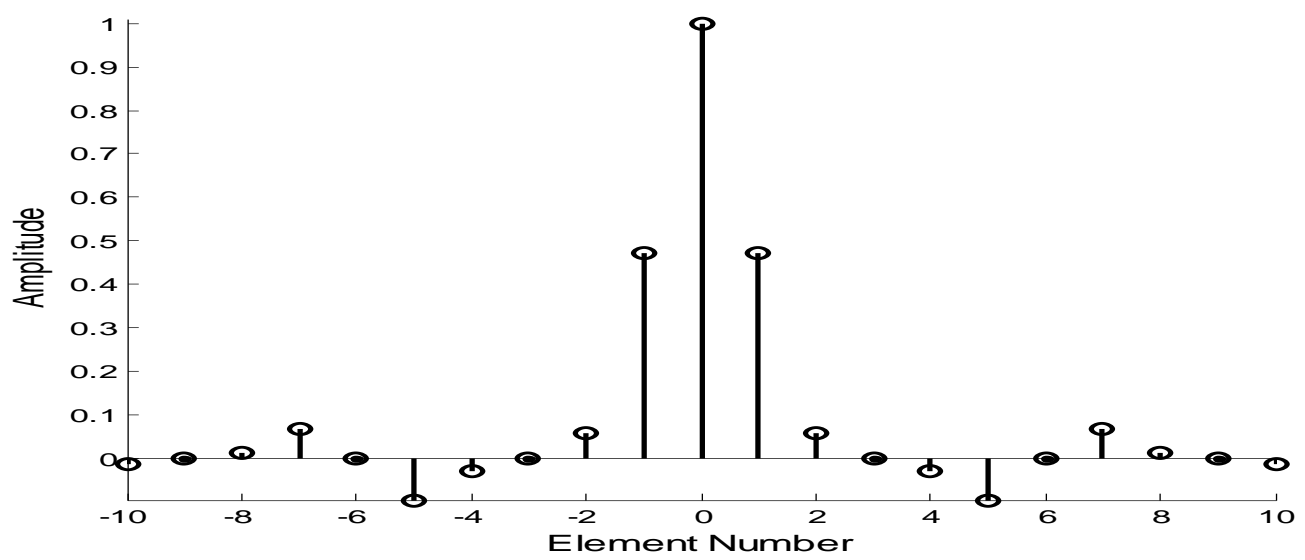

Fig.2.Amplitude distribution of central sub array for $\mathrm{N}=21$

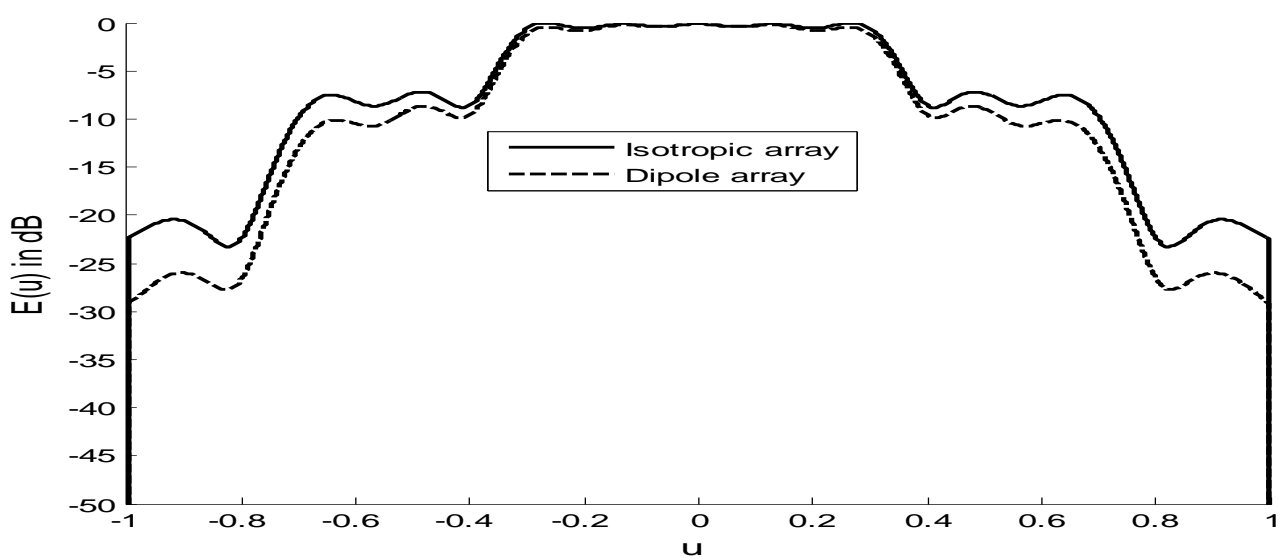

Fig.3.Radiation pattern of central sub array for $\mathrm{N}=21$

Table.1.Excitation levels of 21 elements for Three Stair-step pattern

\begin{tabular}{cc}
\hline Element number & Amplitude level \\
\hline-10 & -0.0118 \\
-9 & 0.0000 \\
-8 & 0.0148 \\
-7 & 0.0675 \\
-6 & -0.0000 \\
-5 & -0.0945 \\
-4 & -0.0295 \\
-3 & 0.0000
\end{tabular}




\begin{tabular}{cc}
-2 & 0.0591 \\
-1 & 0.4726 \\
0 & 1.0000 \\
1 & 0.4726 \\
2 & 0.0591 \\
3 & 0.0000 \\
4 & -0.0295 \\
5 & -0.0945 \\
6 & -0.0000 \\
7 & 0.0675 \\
8 & 0.0148 \\
9 & 0.0000 \\
10 & -0.0118 \\
\hline
\end{tabular}

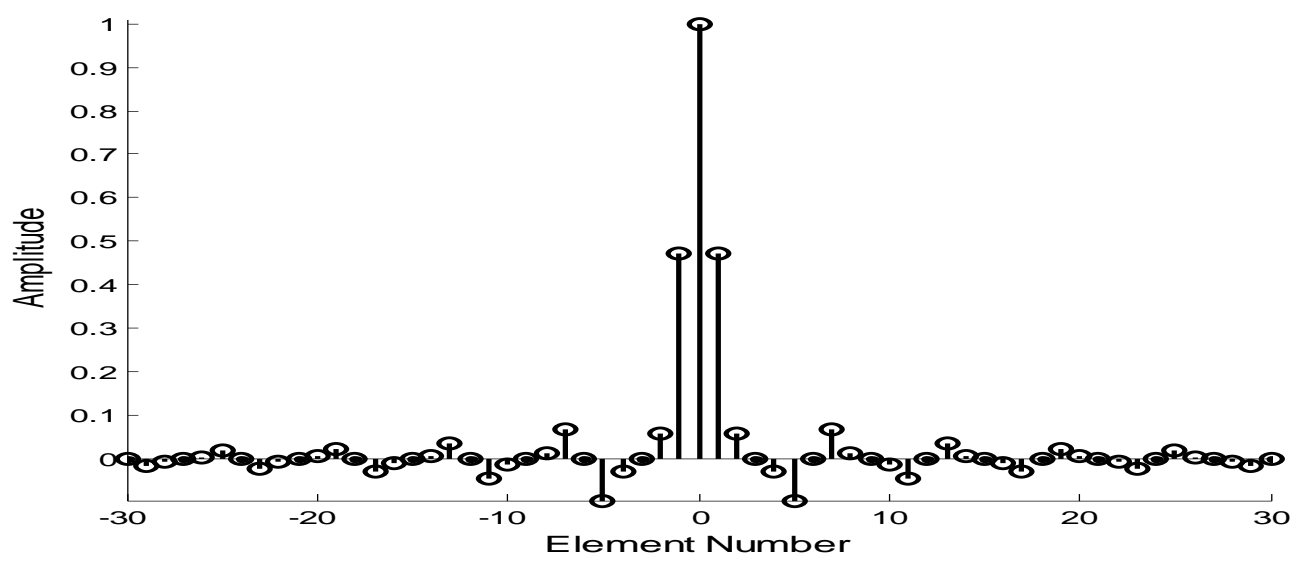

Fig.4.Amplitude distribution of central sub array for $\mathrm{N}=61$

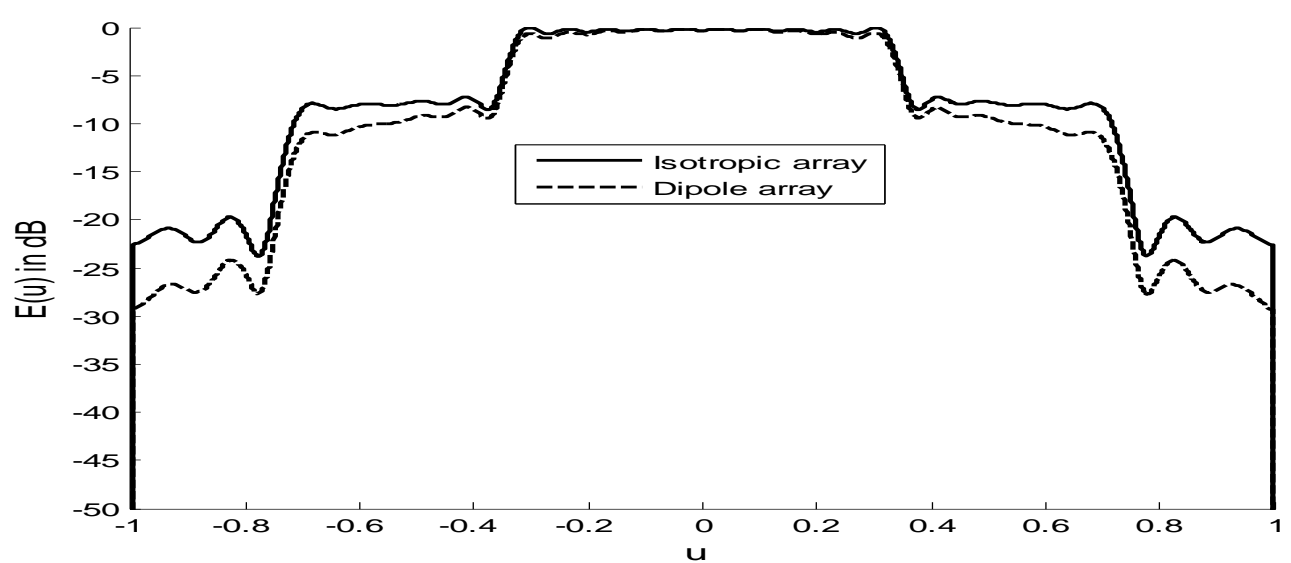

Fig.5.Radiation pattern of central sub array for $\mathrm{N}=61$

Table.2.Excitation levels of 61 elements for Three Stair-step pattern.

\begin{tabular}{cccc}
\hline Element number & Amplitude level & Element number & Amplitude level \\
\hline-30 & -0.0000 & 1 & 0.4726 \\
-29 & -0.0163 & 2 & 0.0591 \\
-28 & -0.0042 & 3 & 0.0000 \\
-27 & 0.0000 & 4 & -0.0295 \\
-26 & 0.0045 & 5 & -0.0945 \\
-25 & 0.0189 & 6 & -0.0000 \\
-24 & -0.0000 & 7 & 0.0675 \\
-23 & -0.0205 & 8 & 0.0148 \\
-22 & -0.0054 & 9 & 0.0000 \\
-21 & -0.0000 & 10 & -0.0118 \\
-20 & 0.0059 & 11 & -0.0430 \\
-19 & 0.0249 & 12 & -0.0000 \\
-18 & -0.0000 & 13 & 0.0364 \\
-17 & -0.0278 & 14 & 0.0084
\end{tabular}




$\begin{array}{ccccc}-16 & -0.0074 & 15 & 0.0000 \\ -15 & 0.0000 & 16 & -0.0074 \\ -14 & 0.0084 & 17 & -0.0278 \\ -13 & 0.0364 & 18 & -0.0000 \\ -12 & -0.0000 & 20 & 0.0249 \\ -11 & -0.0430 & 21 & -0.0059 \\ -10 & -0.0118 & 22 & -0.0054 \\ -9 & 0.0000 & 23 & -0.0205 \\ -8 & 0.0148 & 24 & -0.0000 \\ -7 & 0.0675 & 25 & 0.0189 \\ -6 & -0.0000 & 26 & 0.0045 \\ -5 & -0.0945 & 27 & 0.0000 \\ -4 & -0.0295 & 28 & -0.0042 \\ -3 & 0.0000 & 29 & -0.0163 \\ -1 & 0.0591 & 30 & -0.0000 \\ 0\end{array}$

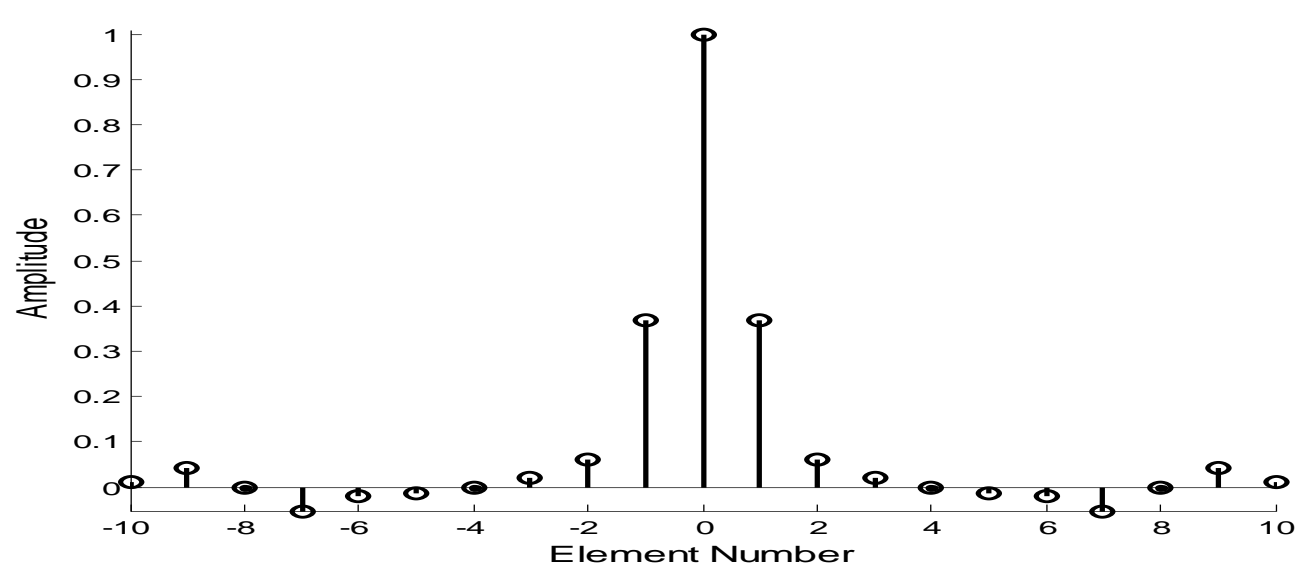

Fig.6.Amplitude distribution of central sub array for $\mathrm{N}=21$

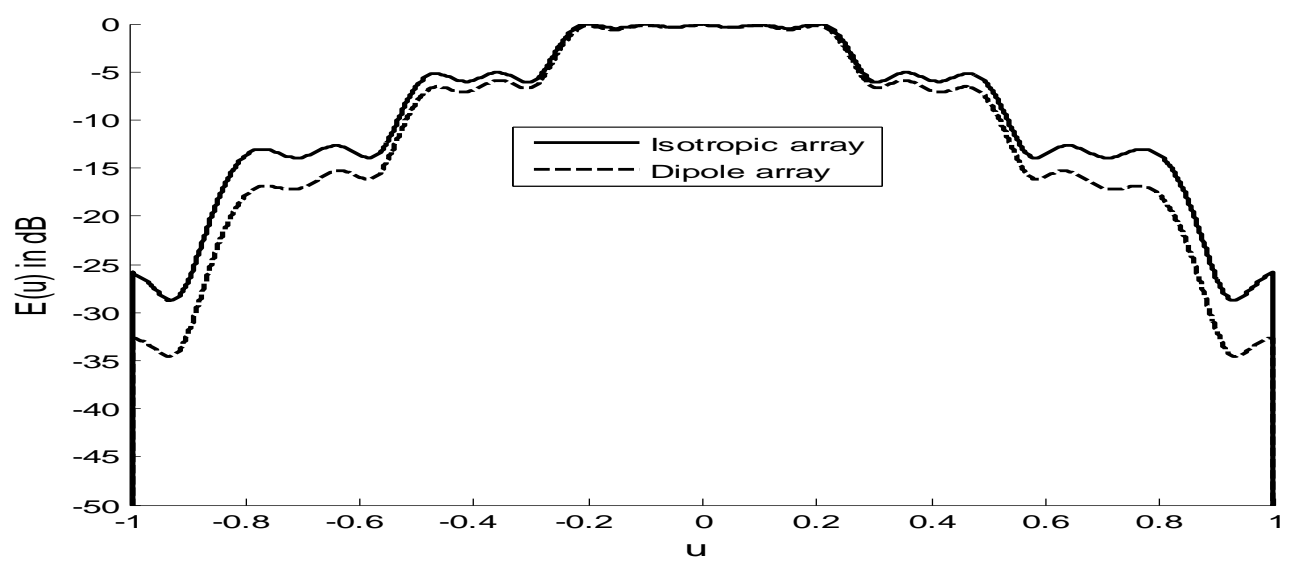

Fig.7.Radiation pattern of central sub array for $\mathrm{N}=21$

Table.3.Excitation levels of 21 elements for Four Stair-step pattern.

\begin{tabular}{cc}
\hline Element number & Amplitude level \\
\hline-10 & 0.0122 \\
-9 & 0.0410 \\
-8 & -0.0000 \\
-7 & -0.0527 \\
-6 & -0.0204 \\
-5 & -0.0127 \\
-4 & 0.0000 \\
-3 & 0.0211 \\
-2 & 0.0611 \\
-1 & 0.3689 \\
0 & 1.0000 \\
1 & 0.3689 \\
2 & 0.0611 \\
3 & 0.0211
\end{tabular}




\begin{tabular}{cc}
4 & 0.0000 \\
5 & -0.0127 \\
6 & -0.0204 \\
7 & -0.0527 \\
8 & -0.0000 \\
9 & 0.0410 \\
10 & 0.0122 \\
\hline
\end{tabular}

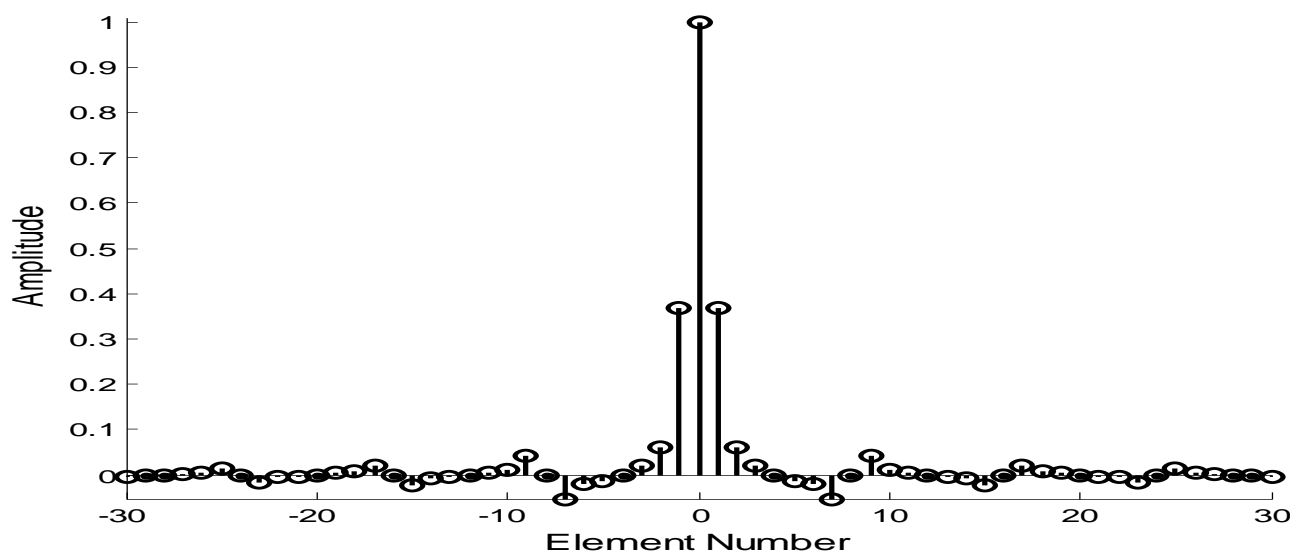

Fig.8.Amplitude distribution of central sub array for $\mathrm{N}=61$

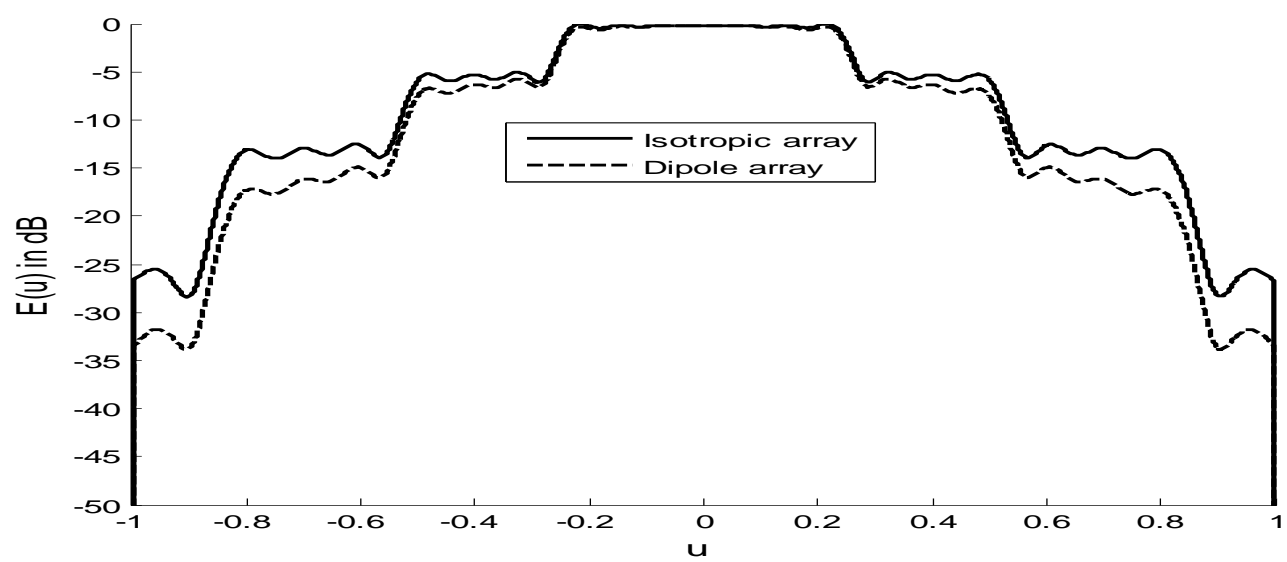

Fig.9.Radiation pattern of central sub array for $\mathrm{N}=61$

Table.4.Excitation levels of 61 elements for Four Stair-step pattern.

\begin{tabular}{cccc}
\hline $\begin{array}{c}\text { Element } \\
\text { number }\end{array}$ & Amplitude level & Element number & Amplitude level \\
\hline-30 & -0.0041 & 1 & 0.3689 \\
-29 & -0.0022 & 2 & 0.0611 \\
-28 & -0.0000 & 3 & 0.0211 \\
-27 & 0.0023 & 4 & 0.0000 \\
-26 & 0.0047 & 5 & -0.0127 \\
-25 & 0.0148 & 6 & -0.0204 \\
-24 & -0.0000 & 7 & -0.0527 \\
-23 & -0.0160 & 8 & -0.0000 \\
-22 & -0.0056 & 9 & 0.0410 \\
-21 & -0.0030 & 10 & 0.0122 \\
-20 & 0.0000 & 11 & 0.0058 \\
-19 & 0.0033 & 12 & 0.0000 \\
-18 & 0.0068 & 13 & -0.0049 \\
-17 & 0.0217 & 14 & -0.0087 \\
-16 & -0.0000 & 15 & -0.0246 \\
-15 & -0.0246 & 16 & -0.0000 \\
-14 & -0.0087 & 17 & 0.0217 \\
-13 & -0.0049 & 18 & 0.0068 \\
-12 & 0.0000 & 19 & 0.0033 \\
-11 & 0.0058 & 20 & 0.0000 \\
-10 & 0.0122 & 21 & -0.0030 \\
\hline
\end{tabular}




\begin{tabular}{lccc}
-9 & 0.0410 & 22 & -0.0056 \\
-8 & -0.0000 & 23 & -0.0160 \\
-7 & -0.0527 & 24 & -0.0000 \\
-6 & -0.0204 & 25 & 0.0148 \\
-5 & -0.0127 & 26 & 0.0047 \\
-4 & 0.0000 & 27 & 0.0023 \\
-3 & 0.0211 & 28 & -0.0000 \\
-2 & 0.0611 & 29 & -0.0022 \\
-1 & 0.3689 & 30 & -0.0041 \\
0 & 1.0000 & & \\
\hline
\end{tabular}

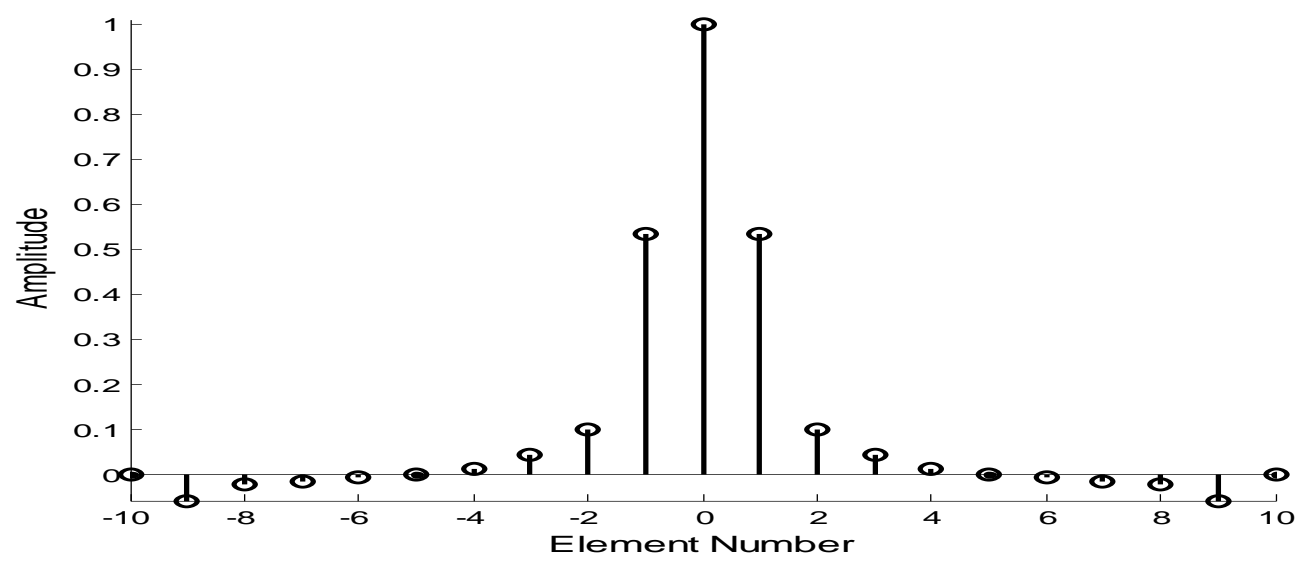

Fig.10.Amplitude distribution of central sub array for $\mathrm{N}=21$

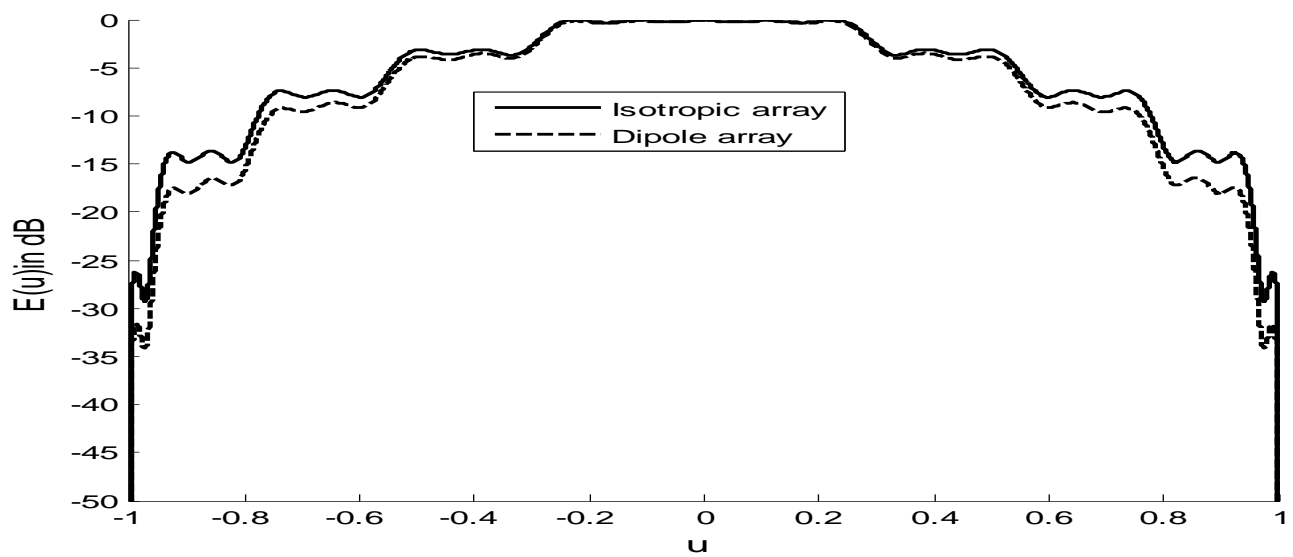

Fig.11.Radiation pattern of central sub array for $\mathrm{N}=21$

Table.5.Excitation levels of 21 elements for Five Stair-step pattern .

\begin{tabular}{cc}
\hline Element number & Amplitude level \\
\hline-10 & -0.0000 \\
-9 & -0.0594 \\
-8 & -0.0249 \\
-7 & -0.0180 \\
-6 & -0.0078 \\
-5 & 0.0000 \\
-4 & 0.0118 \\
-3 & 0.0420 \\
-2 & 0.0996 \\
-1 & 0.5344 \\
0 & 1.0000 \\
1 & 0.5344 \\
2 & 0.0996 \\
3 & 0.0420 \\
4 & 0.0118 \\
5 & 0.0000 \\
6 & -0.0078 \\
7 & -0.0180 \\
8 & -0.0249 \\
9 & -0.0594 \\
10 & -0.0000 \\
\hline
\end{tabular}




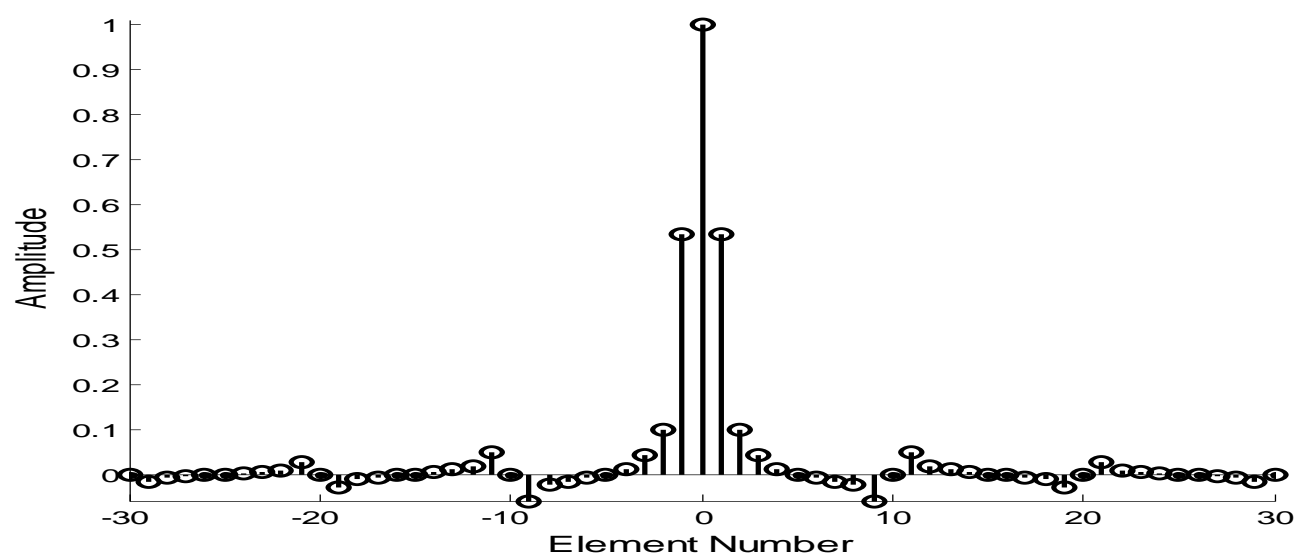

Fig.12.Amplitude distribution of central sub array for $\mathrm{N}=61$

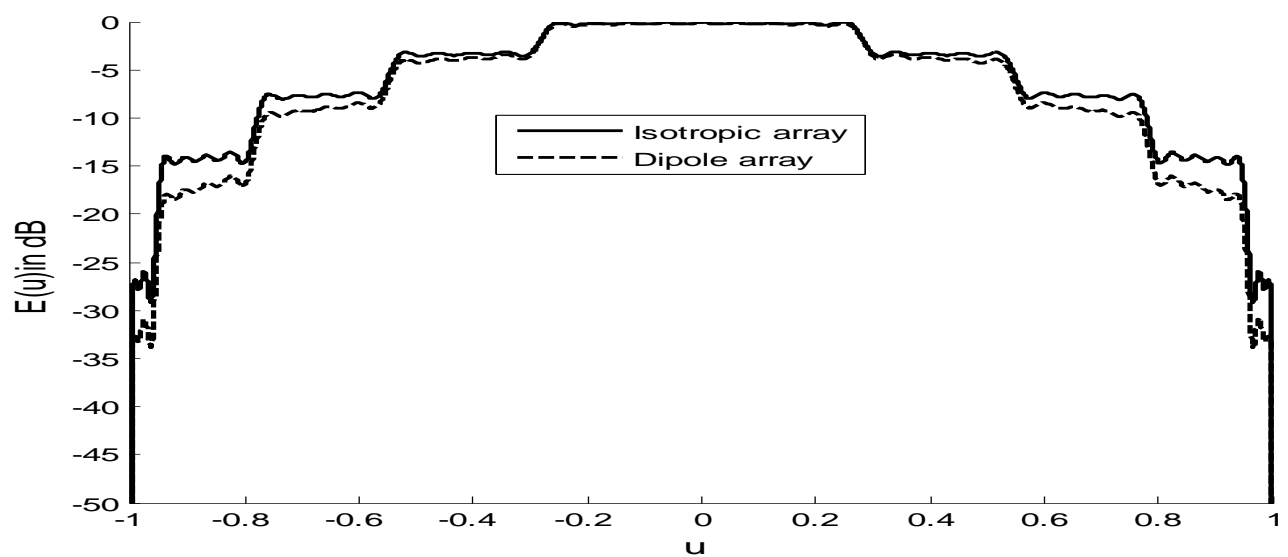

Fig.13.Radiation pattern of central sub array for $\mathrm{N}=61$

Table.6.Excitation levels of 61 elements for Five Stair-step pattern.

\begin{tabular}{|c|c|c|c|}
\hline Element number & Amplitude level & Element number & Amplitude level \\
\hline-30 & -0.0000 & 1 & 0.5344 \\
\hline-29 & -0.0184 & 2 & 0.0996 \\
\hline-28 & -0.0071 & 3 & 0.0420 \\
\hline-27 & -0.0047 & 4 & 0.0118 \\
\hline-26 & -0.0018 & 5 & 0.0000 \\
\hline-25 & 0.0000 & 6 & -0.0078 \\
\hline-24 & 0.0020 & 7 & -0.0180 \\
\hline-23 & 0.0055 & 8 & -0.0249 \\
\hline-22 & 0.0091 & 9 & -0.0594 \\
\hline-21 & 0.0254 & 10 & -0.0000 \\
\hline-20 & -0.0000 & 11 & 0.0486 \\
\hline-19 & -0.0281 & 12 & 0.0166 \\
\hline-18 & -0.0111 & 13 & 0.0097 \\
\hline-17 & -0.0074 & 14 & 0.0034 \\
\hline-16 & -0.0029 & 15 & 0.0000 \\
\hline-15 & 0.0000 & 16 & -0.0029 \\
\hline-14 & 0.0034 & 17 & -0.0074 \\
\hline-13 & 0.0097 & 18 & -0.0111 \\
\hline-12 & 0.0166 & 19 & -0.0281 \\
\hline-11 & 0.0486 & 20 & -0.0000 \\
\hline-10 & -0.0000 & 21 & 0.0254 \\
\hline-9 & -0.0594 & 22 & 0.0091 \\
\hline-8 & -0.0249 & 23 & 0.0055 \\
\hline-7 & -0.0180 & 24 & 0.0020 \\
\hline-6 & -0.0078 & 25 & 0.0000 \\
\hline-5 & 0.0000 & 26 & -0.0018 \\
\hline-4 & 0.0118 & 27 & -0.0047 \\
\hline-3 & 0.0420 & 28 & -0.0071 \\
\hline-2 & 0.0996 & 29 & -0.0184 \\
\hline-1 & 0.5344 & 30 & -0.0000 \\
\hline 0 & 1.0000 & & \\
\hline
\end{tabular}




\section{Conclusion}

In this work, we proposed a new amplitude distribution for a array, patterns are computed for different stair steps. Using Fourier transform method, stair step patterns are produced from array of dipoles. The results are compared with those of array of isotropic radiators. It is clear from the computed patterns, there exists considerable deviation in the patterns of isotropic and dipole arrays. These patterns are intensively used to identify more than one target moving in different altitudes and different angular regions.

\section{References}

[1]. C.A.Balanis, “Antenna Theory Analysis and Design,” John Wiley and sons, Inc., United States of America, 1982

[2]. G.S.N.Raju, "Antennas and Propagation", Pearson Education, 2005.

[3]. W. L. Stutzman and G. A. Thiele, "Antenna Theory and Design”, John Wiley \& Sons Inc., U. S. A.

[4]. Tse-Tong Chia and Wai-Yean Lim, "Design of low profile cylindrical Lune burg lens Antenna" IEEE Trans. Antennas Propagat. vol. 12, No.6,Sec.2009.

[5]. S.Jain and R.Mittra, "Broadband Flat-base Lune burg lens Antenna for wide angle scan" IEEE Trans. Antennas Propagat. June.2014.

[6]. R.K. Luneburg, Mathematical Theory of optics. Berkeley and Los Angeles, CA:University of California Press,1964.

[7]. Mosallaei, H.and Rahmat-Samii, Y., "Non-uniform Luneburg lens antennas: a design approach based on genetic algorithms," Antennas and Propagation Society International Symposium, IEEE , vol.1, no., pp.434,437 vol.1, 11-16,July1999.

[8]. G.V.Borgiotti, "Degrees of freedom of an antenna scanned in a limited sector", in IEEE Antennas Propagat. Symp. Dig. June 1975, p. 319

[9]. E.C.Dufort, "Optimum Optical limited Scan Antenna”, IEEE Trans. Antennas Propagate. vol. AP-34, no.9, Sept. 1986.

[10]. A. Sudhakar and G.S.N.Raju, "Generation of Stair-Step Radiation Patterns of an Array of Antenna", AMSE Journal, France.

[11]. Kang Wook Kim, "Characterizations of Spherical Luneburg Lens Antennas with Air-gaps and Dielectric Losses", Journal of the Korea Electromagnetic Engineering Society, vol.1.No.1, May 2001.

[12]. W.Rotman and R. F. Turner, "Wide angle microwave lens for line source applications", IEEE Trans. Antennas Propagat. vol. AP11, p. 623, Nov. 1963.

[13]. R.J.Mailloux and P. Blacksmith, “Array and reflector techniques for airport precision approach radars”, Microwave J., vol. 17, p. 35 , Oct. 1974

[14]. J.F.A.Ormsby, “ Antenna, Load and Field effects on the bastatic scattering patterns from a linear dipole array ",IEEE Trans. Antennas Propagat. vol. AP-27, No.1,Jan.1979.

[15]. Tai, C.T. “Dipoles and Monopoles”, ch.4 in Antenna Engineering Hand Book, R.C. Johnson and H.Jasik. Eds, New York; McGraw-Hill, 1984.

[16]. J.C. Bregains and F.Ares, "Variation in bandwidths among solutions to shaped beam synthesis problems concerning linear arrays of parallel dipoles",IEEE Trans. Antennas Propagat. vol. 53, No.1,Jan.2005.

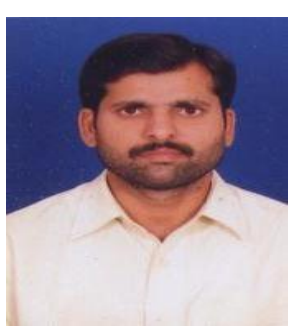

M.Chandrasekhar received his B.E. in Electronics and Communication Engineering in the year of 2002 from Andhra University and the Master of Technology in Radar and Microwave Engineering in 2007 from Andhra University College of Engineering (A). Currently, he is working towards his Ph.D degree in the department of Electronics and Communication Engineering, Andhra University College of Engineering (A). His Research interests include Array Antennas, EMI/EMC and Soft Computing. He is a life member of SEMCE (India).

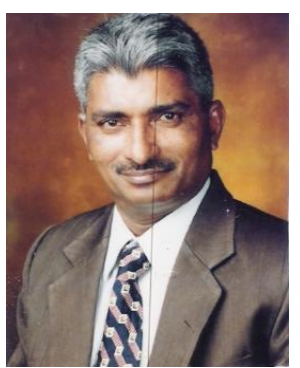

Dr. G.S.N. Raju received his B.E., M.E. with distinction and first rank from Andhra University and Ph.D. from IIT, Kharagpur. At present, he is the Vice - Chancellor of Andhra University and a Senior Professor in Electronics and Communication Engineering. He is in teaching and research for the last 30 years in Andhra University. He guided 28 Ph.D.s in the fields of Antennas, Electromagnetics, EMI/EMC and Microwave, Radar Communications, Electronic circuits. Published about 304 technical papers in National/ International Journals/ Conference Journals and transactions. He is the recipient of 'The State Best Teacher Award' from the Government of Andhra Pradesh in 1999, 'The Best Researcher Award' in 1994, 'Prof. Aiya Memorial National IETE Award' for his best Research guidance in 2008 and Dr. Sarvepalli Radhakrishnan Award for the Best Academician of the year 2007. He was a visiting Professor in the University of Paderborn and also in the University Karlsruhe, Germany in 1994. He held the positions of Principal, Andhra University College of Engineering (A), Visakhapatnam, Chief Editor of National Journal of Electromagnetic Compatibility. Prof. Raju has published five textbooks Antennas and Wave Propagation, Electromagnetic Field Theory and Transmission Lines, Electronics Devices and Circuits, Microwave Engineering, Radar Engineering and Navigational Aids. Prof. Raju has been the best faculty performer in Andhra University with the performance index of $99.37 \%$. 